\title{
Combinational concentration gradient confinement through stagnation flow $\dagger$
}

Toh G. G. Alicia, ${ }^{a, b}$ Chun Yang, ${ }^{* a}$ Zhiping Wang, ${ }^{b}$ and Nam-Trung Nguyen ${ }^{*}$

\begin{abstract}
Concentration gradient generation in microfluidics is typically constrained by two conflicting mass transport requirements: short characteristic times $(\tau)$ for precise temporal control of concentration gradients but at the expense of high flow rates and hence, high flow shear stresses $(\sigma)$. To decouple the limitations from these parameters, here we propose the use of stagnation flows to confine concentration gradients within large velocity gradients that surround the stagnation point. We developed a modified cross-slot (MCS) device capable of feeding binary and combinational concentration sources in stagnation flows. We show that across the velocity well, source-sink pairs can form permanent concentration gradients. As source- sink concentration pairs are continuously supplied to the MCS, a permanently stable concentration gradient can be generated. Tuning the flow rates directly controls the velocity gradients, and hence the stagnation point location, allowing the confined concentration gradient to be focused. In addition, the flow rate ratio within the MCS rapidly controls ( $\tau \sim 50 \mathrm{~ms}$ ) the location of the stagnation point and the confined combinational concentration gradients at low flow shear (0.2 $\mathrm{Pa}<\sigma<2.9 \mathrm{~Pa}$ ). The MCS device described in this study establishes the method for using stagnation flows to rapidly generate and position low shear combinational concentration gradients for shear sensitive biological assays.
\end{abstract}

\section{Introduction}

Stagnation flows, and their control have received great attention in the microfluidics community. Recent studies have demonstrated niche applications of benign trapping and manipulation of single cells ${ }^{1,2}$, biomolecules $^{3-5}$ and particles ${ }^{6-8}$ in hydrodynamically formed stagnation flows. Stagnation flows are flow fields that surround stagnation points- singularities with zero local velocities. ${ }^{9}$ An early demonstration of stagnation flow was performed in a four-roll mill apparatus developed by G. I. Taylor in $1934 .^{10}$ In microfluidics, the underlying principle behind stagnation flows is readily exploited due to the steady nature of Newtonian fluids in the laminar flow regime. ${ }^{11-13}$ One of the easiest methods to generate stagnation flow in microfluidics is the formation of planar extensional flows. ${ }^{3,5,9,14}$ Most commonly, this type of flow is manifested in the presence of opposing flow streams with equal velocity field strength; the interface that forms between the opposing streams intersects the axis of flow at the stagnation point. Stagnation points of extensional flows have been demonstrated in microfluidic cross-slot configurations. ${ }^{7,11,14}$ 
Stagnation flows in microfluidic cross-slot devices have been used to confine and stretch individual strands of DNA. ${ }^{4}$ Perkins et al. utilized the homogeneous velocity gradients surrounding a stagnation point to study the non-Newtonian polymer dynamics of coiled and stretched DNA strands. ${ }^{4}$ Recently, JohnsonChavarria et al. demonstrated that a cross-slot device can be used to hydrodynamically trap individual $E$. coli bacteria for approximately two hours. ${ }^{1}$ Although recent efforts have focused on the use of stagnation flow to confine micro/ nano- particles, the potential of stagnation flows to confine and control concentration gradients has not been fully explored. Combinational concentration gradients can be formed by protracting flows of multiple chemical species across the large velocity gradients surrounding stagnation points. In the case of a stagnation flow, chemical concentrations can be fed continuously across the stagnation point to produce perpetually stable concentration gradients that can be moved in an instance by controlling inlet flow rates. Thus far, most microfluidic gradient generators have relied on higher flow rates to lower the characteristic times for dynamically controlling concentration gradients. ${ }^{15-17}$ However, high flow rates and hence, high flow velocities, significantly increase the flow shear effects within the device. ${ }^{18-20}$ Device modifications such as expansion chambers ${ }^{21}$, hydraulic resistance networks ${ }^{22}$, porous gels ${ }^{23}$ and membranes ${ }^{24}$ were necessary to reduce shear effects in such gradient generators. Chambers with steep depth expansions were designed to decrease flow velocities from inlet channels while hydrogels ${ }^{23}$ or membranes ${ }^{24}$ add tortuosity to the inflow path, thereby achieving similar velocity and shear reduction effects. Notwithstanding shear effects, these additions also dramatically increase the critical time required to stabilize or spatially move steady-state concentration gradients. In this respect, the utilization of stagnation flows is a novel approach to rapidly generate concentration gradients while limiting flow shear effects due to the diminishing velocity fields surrounding the stagnation point.

In this paper, we introduce a new class of gradient generators that utilize stagnation flows to confine binary or combinational concentration gradients within the large velocity gradients of a stagnation point. The gradient generator is a modified cross-slot (MCS) device that allows gradient landscapes to be confined, focused and moved by hydrodynamic adjustments in stagnation flow. We characterize the stagnation flow within the MCS in terms of key parameters such as velocity gradient, length and hydrodynamic localization of the concentration gradients. In addition, we compare the experimental data to numerical simulation results of the stagnation flow within a MCS. Based on these investigations, we demonstrate the use of a MCS device to generate perpetual source-sink diffusion profiles. Unlike conventional microfluidic gradient generators, our device can simultaneously suppress flow shear effects and dynamically as well as spatially control binary or combinational concentration gradients. 


\section{Methods and materials}

\section{Modified cross-slot device design and fabrication}

Fig. 1A shows a microscope image of the modified cross-slot (MCS) device for concentration gradient generation using a stagnant flow. The device consists of two layers, a fluidic layer and a pneumatic valve control layer. The fluidic layer was designed as a MCS with delivery channels (of $200 \mu \mathrm{m}$ width and $12 \mu \mathrm{m}$ depth). The delivery channels are connected with diffusion channels (of $30 \mu \mathrm{m}$ width and $12 \mu \mathrm{m}$ depth). These channels introduce the solutes into a $1 \mathrm{~mm}$ by $1 \mathrm{~mm}$ square stagnation flow chamber (see Fig. 1A). Both device layers were fabricated using soft lithography with a micromachined silicon mold and polydimethylsiloxane (PDMS) casting. ${ }^{25}$ The alignment and bonding between the fluidic layer and the valve control layer were achieved by following the fabrication processes of membrane- based 'Quake valves' ${ }^{26,27}$ Briefly, the pneumatic valve control layer was formed via spin coating and curing a thin layer of PDMS ( $\sim 5 \mu \mathrm{m}$ thick) over the valve layer features that are $30 \mu \mathrm{m}$ in height, thereby forming a $15 \mu \mathrm{m}$ thick membrane. The cured valve layer was subsequently aligned and bonded to the fluidic layer. The orthogonal intersection between channels in the fluid layer and valve control layer form a 'push-up' valve ${ }^{27}$, as shown in Fig. 1A, C.

\section{Stagnation flow and concentration gradient validation}

Stagnation flow within the device was validated through the use of carboxylated fluorescent tracer beads (2 $\mu \mathrm{m}$ diameter, Polybead, Polysciences, Inc.). The tracer beads were added to deionized water at a concentration of $1 \times 10^{4}$ beads $\mathrm{ml}^{-1}$; bead concentrations between $1 \times 10^{3}$ to $1 \times 10^{4}$ beads $\mathrm{ml}^{-1}$ were found to produce optimal tracing images with minimal occurrence of bead aggregation. Streakline images of stagnation flow (Fig. 1B) were obtained by digitally superimposing the trajectory of beads across 200 sequential images (recorded at a time intervals of 30ms) via ImageJ software (National Institutes of Health Maryland, USA). ${ }^{28}$ Images tracing bead movement had a size of 1048 by 1048 pixels and a pixel resolution of $1.136 \mu \mathrm{m} /$ pixel. Quantitative mapping of flow velocities within the device was performed by correlating image pairs that were captured at a time interval of 30ms. Digital correlation of the images was conducted by a Matlab open source particle image velocimetry (PIV) software. $^{29}$

In concentration gradient experiments, binary concentration gradient profiles were quantified through the use of $1 \mathrm{~m} \underline{\mathrm{M}}$ fluorescein solutions. In the case of combinational concentration gradients, concentration profiles were quantified using $1 \mathrm{~m} \underline{\mathrm{M}}$ of fluorescein and $0.1 \mathrm{~m} \underline{\mathrm{M}}$ of Rhodamine 6G solutions. A lower concentration of Rhodamine 6G was used to prevent leeching of the dye into the PDMS device. ${ }^{30}$ 
Fluorescein dye solutions have an excitation wavelength of 470nm and an emission wavelength of 520nm. Rhodamine 6G dye solutions have an excitation wavelength of 480nm and an emission wavelength of $580 \mathrm{~nm}$. In order to normalize the actual dye concentration to the intensity of the emitted wavelength, the fluorescence emission of each dye was first calibrated using a spectrophotometer (Nanodrop 3300, Thermo Fisher Scientific). The fluorescence intensity emitted by both dyes had a linear relationship to dye concentration (see supplementary Fig. S1), indicating that actual dye concentrations could be directly derived from the normalized intensity measurements of concentration gradients. Concentration gradients were established by pressure controlled perfusion into the four flow inlets. A customized pressure perfusion system maintained stable liquid flow in the device through volume displacement by pressurized air (see Fig. 1C). Initial attempts in controlling stagnation flow with syringe pumps were unsuccessful due to nontandem flow pulsations (resulting from asynchronous syringe motor stepping) between the four flow inlets during perfusion. ${ }^{31}$ On the other hand, the pressure pump configuration shown in Fig. 1C allowed precise and pulse-free control of injection flow rates ranging from $100 \mathrm{nl} \mathrm{s} \mathrm{s}^{-1}$ to $1000 \mathrm{nl} \mathrm{s} \mathrm{s}^{-1}$. Adjustments in pump pressure resulted in changes to the device flow rate. A calibration of pump pressures to device flow rate was performed using a flow rate sensor (Mitos flow rate sensor, Dolomite) (supplementary Fig. S2). Hydrodynamic adjustments to the position of the stagnation point was achieved by actuating the on-chip membrane valves shown in Fig. 1C (labeled as $V_{1}$ to $V_{4}$ ). Both the valve and inlet flow pressures could be pre-programmed with respect to time (see graphical schematic in Fig. 1C) via a digital user interface. The user interface was created using LabVIEW and controlled the solenoid valves, pressure actuators and regulators through an NI DAQ module. On-chip valves enable fast and direct positioning of the stagnation point (and the corresponding concentration gradients) by reducing the flow rates entering the stagnation chamber. For example, when the injection flow rate, $Q$ was set at $100 \mathrm{nl} \mathrm{s}{ }^{-1}$, a valve actuation pressure of $16 \mathrm{kPa}$ resulted in a chamber flow rate of $50 \mathrm{nl} \mathrm{s}^{-1}$ (50\% valve closure). Flow rates entering the main chamber were calibrated based on the percentage of valve closure; this was derived from outlet flow rate measurements before and after the valve was actuated (shown in supplementary Fig. S3). On-chip valves are able to control flow rates within approximately $20 \mathrm{~ms}$.

\section{Numerical simulation}

The governing equations of the steady two-dimensional Stokes equation and the steady convection-diffusion equation were numerically solved using the finite element simulation software, Comsol Multiphysics 4.2. Incompressible Newtonian fluid properties of water (density- $998 \mathrm{~kg} \mathrm{~m}^{-3}$; dynamic viscosity- $8.9 \times 10^{-4}$ Pa.s) were used to simulate the flow field in the device. In binary gradient simulations, a diffusion coefficient of fluorescein in water, $D_{\text {fluor }}=5 \times 10^{-10} \mathrm{~m}^{2} \mathrm{~s}^{-1}$ was used, based on previously reported values. ${ }^{32}$ In combinational 
gradient simulations, a diffusion coefficient of Rhodamine $6 \mathrm{G}$ in water, $\mathrm{D}_{\mathrm{Rho6G}}=4 \times 10^{-10} \mathrm{~m}^{2} \mathrm{~s}^{-1}$ was used. ${ }^{33}$ No-slip boundary conditions were applied to the perimeter of the device geometry, except at the four inlets or outlets. Inlet flow boundary conditions were specified by given flow rates ranging from $100 \mathrm{nl} \mathrm{s}^{-1}$ to 1000 $n \mathrm{l} \mathrm{s}^{-1}$, while outlet boundary conditions were set to ambient pressure. In concentration gradient simulations, the perimeter of the device geometry, except for the inlets and outlets were defined as zero mass flux penetration boundaries.

\section{Results and discussions}

\section{Stagnation flow in the modified cross-slot device}

In the modified cross-slot (MCS) device, a stagnation point is generated by planar extensional flows resulting from four opposing flow streams that converge in the chamber. The MCS is modified from the conventional cross-slot device ${ }^{34}$ with the addition of diffusion channels that direct flow into the stagnation flow zone. The optimized narrow diffusion channels increase flow resistance and also act as flow velocity buffers to reduce shear effects within the main chamber. This design allows the introduction of dual sourcesink concentration pairs into the main stagnation chamber, thereby facilitating the generation of overlapping gradients for a maximum of four different chemical species, which to our best knowledge has not been reported in the literature before. Under symmetrical flow conditions, i.e. $Q=Q_{1}=Q_{2}=Q_{3}=Q_{4}$, the MCS, similar to conventional cross-slot devices, generates a stagnation point at the intersection of the four converging flows. Bead movements shown in Fig. 1B trace a group of hyperbolic streaklines surrounding the stagnation point at the centre of the chamber. PIV mapping of flow velocities in the stagnation chamber revealed a velocity gradient surrounding the central stagnation point (Fig. 2A). Near the stagnation point, the velocity potential and the velocity vector field are analogous to stagnation flow confined by corner geometries: ${ }^{6}$

$$
\begin{aligned}
& \varphi(x, y)=\frac{\dot{\varepsilon}}{2}\left(x^{2}-y^{2}\right) \\
& v_{x}=\frac{\partial \varphi}{\partial x}=\dot{\varepsilon} x ; v_{y}=\frac{\partial \varphi}{\partial y}=-\dot{\varepsilon} y
\end{aligned}
$$

where $x$ and $y$ are Cartesian co-ordinates corresponding to the reference frame shown in Fig. $2 \mathbf{A}$ and, $v_{x}$ and $v_{y}$ are the velocity components along the $x$ and $y$ direction, respectively. Based on the co-ordinate system defined, $\dot{\varepsilon} \equiv \partial v_{x} / \partial x \equiv-\partial v_{y} / \partial y$ where $\dot{\varepsilon}$ is the flow strain rate that defines the steepness of the velocity well surrounding the stagnation point.

As theoretically predicted, point analysis of equation (1) confirms the presence of a stagnation point, i.e. a 
velocity minima, at the chamber centre such that: ${ }^{9}$

$\left.v_{x}\right|_{x=0}=\left.\frac{\partial \varphi}{\partial x}\right|_{x=0}=0 ;\left.v_{y}\right|_{y=0}=-\left.\frac{\partial \varphi}{\partial y}\right|_{y=0}=0$

The PIV measurements revealed stagnation flow that was characterized by a linear and homogenous flow strain rate, $\dot{\varepsilon}$, within the vicinity of the stagnation point (shown in Fig. 2B). Within $-400 \mu \mathrm{m} \leq x \leq 400 \mu \mathrm{m}$, a linear flow strain rate, $\dot{\varepsilon}$, could be directly measured from the velocity profile such that $\dot{\varepsilon} \equiv \frac{\partial v_{x}}{\partial x}$ (see inset in Fig. 2B). The flow strain rate was observed to increase with flow rates such that $\dot{\varepsilon}=f(Q)=0.0198 \cdot Q$ (see supplementary Fig. S4). The empirical relationship between $\dot{\varepsilon}$ and $Q$ is indicative of strain rate properties for Newtonian fluids experiencing stable stagnation flows within the laminar flow regime. ${ }^{14,34}$ Deriving the velocity magnitude, $U$, from the individual velocity components, $v_{x}$ and $v_{y}$, we found that a velocity gradient (velocity well) could be formed around the stagnation point. The velocity magnitudes that were measured along line A'-A" and plotted in Fig. 2C highlight the presence of a velocity well. Data plotted from $Q=100 \mathrm{nl} \mathrm{s}^{-1}$ to $Q=1000 \mathrm{nl} \mathrm{s}^{-1}$ showed that increases in flow rates resulted in the formation of steeper velocity wells. Experimental data obtained for velocity magnitudes (Fig. 2C) match closely with the velocity profiles that were numerically mapped from equations $(1,2)$. Theoretically, a stagnation point is a singularity in which the local fluid velocity is zero, i.e. $v(x, y)=0$. Experimentally, however, it was not physically possible to measure exact zero fluid velocities since empirical velocity measurements were derived from bead trajectory and movement. In our experiments, we considered the stagnation point as a point where local velocities approached zero, i.e. $v(x, y=0) \rightarrow 0$ such that the flow velocities at the stagnation point were approximately 4-5 orders of magnitude smaller than the maximum velocities within the chamber. Two-dimensional flow velocity mapping near the stagnation point reveals an inverted conical velocity well surrounding the stagnation point (see Fig. 2D). It is anticipated from this data that the steepness of the velocity well, analogous to a potential well, can be used for the confinement of concentration gradients via convective mass transport.

Analysing the maximum flow velocities within the stagnation chamber gives an estimated Reynolds number of $R e=0.13$, for the maximum flow rate of $1000 \mathrm{nl} \mathrm{s} \mathrm{s}^{-1}$. At low $R e$ and smaller flow rates, the maximum shear stress generated within the chamber is expected to be diminishing. Within the stagnation chamber, the shear stress $(\sigma)$ induced by fluid flow can be mapped within the stagnation chamber (see supplementary Fig. S5A). The experimental velocity magnitude $\left(U=\sqrt{v_{x}^{2}+v_{y}^{2}}\right)$ obtained from PIV measurements was used to calculate the wall shear stress. The PIV results were obtained based on the images capturing bead movement at the mid-plane of the device thickness, i.e. $z \sim 6 \mu \mathrm{m}$, since this is the 
location at which the velocity magnitude reaches a maximum value. ${ }^{35}$ Considering Poiseuille's law for the pressure-driven flow, the wall shear stress was calculated as $\tau=6 U_{\text {mean }} \cdot \frac{\mu}{H}$, where $U_{\text {mean }}=\frac{2}{3} U, \mu$ is the fluid's dynamic viscosity and $H$ is the depth of the chamber. We found that the flow rate range of 50nl s${ }^{-1}$ $<Q<1000 \mathrm{nl} \mathrm{s}^{-1}$ induced low in-chamber flow shear stresses ranging from $0.2<\sigma<2.9 P a$ (in supplementary Fig. S5B), indicating that a range of shear stresses could be specifically targeted through flow rate modulations. This finding, along with our earlier findings on velocity wells indicate that stagnation flow could be utilized to augment the mass transport of species, while effectively suppressing flow shear stress effects.

\section{Mass transport of binary concentration gradients}

The use of velocity wells to confine concentration gradients was demonstrated by injecting fluorescein (concentration source) into one of four flow inlets, while the other inlets were injected with water (concentration sinks). Shortly after the flow injection ( $t \sim 50 \mathrm{~ms}$ ), at a flow rate of $Q=100 \mathrm{nl} \mathrm{s} \mathrm{s}^{-1}$, a steady state concentration gradient of fluorescein was formed across the velocity well surrounding the stagnation point (see Fig. 3A). The concentration gradient transported through stagnation flow is steady and non-saturating since the source constantly replenishes concentration species towards the stagnation point (via the convective flow) while the sink removes the concentration species away from the stagnation point (via equal convective flow). In a stagnation flow, the mass transport of concentration species is characterised by the steady-state convection-diffusion equation: ${ }^{9}$

$$
D \vec{\nabla}^{2} C=\vec{\nabla} \varphi \cdot \vec{\nabla} C
$$

where $C$ is the mass concentration of fluorescent dye within the gradient chamber, $D$ is the diffusion coefficient of fluorescein or Rhodamine 6G dye in water and $\varphi=f(\dot{\varepsilon})$ is the velocity potential, described earlier in equation (1).

Physically, equation (4) shows how the steady-state concentration gradient arises due to mass conservation and, the balance between diffusive mass transport $\left(D \vec{\nabla}^{2} C\right)$ and convective mass transport $(\vec{\nabla} \varphi \cdot \vec{\nabla} C)$. Experimentally, upon comparing the concentration gradient profiles (shown in Fig. $\mathbf{3 B}$ ), we found that the concentration gradient length (region) could be stretched or compressed across the velocity well by applying different flow rates. Here, we define the concentration gradient length as the lateral distance between the maximum normalized concentration of 1 and the minimum normalized concentration of 0 . The experimental results obtained were in accordance to the simulation results of the two dimensional concentration contours shown in Fig. 3C. The relationship between the concentration gradient length and the flow rate is presented 
in Fig. 3D. The concentration gradients formed could be elongated by reducing the inlet flowrates. For instance, reducing the flowrate to $50 \mathrm{nl} \mathrm{s}^{-1}$ resulted in gradient lengths of $350 \mu \mathrm{m}$. Alternatively, increasing the flow rate from $50 \mathrm{nl} \mathrm{s}^{-1}$ to $1000 \mathrm{nl} \mathrm{s}^{-1}$ resulted in the compression of gradient lengths from $\sim 350 \mu \mathrm{m}$ to $94 \mu \mathrm{m}$. Both numerical and experimental data follow the same trend such that the gradient length, $L$, is inversely proportional to the flow rate, i.e $L \propto Q^{-1}$. Since our earlier data showed that $\dot{\varepsilon} \propto Q$, the gradient length is therefore inversely related to the steepness of the velocity well where $L \propto \dot{\varepsilon}^{-1}$, proving that velocity gradients surrounding stagnation points can be used to effectively tune concentration gradients. Thus, a reduction in flow rates would further reduce the velocity gradient and in turn, effectively widen the concentration gradient formed. On the other hand, we also found that at Q>800nl s ${ }^{-1}$, the gradient length was limited by a finite mass transfer boundary layer of $\sim 92 \mu \mathrm{m}$. Similar gradient length scales have previously been reported in convection dominated microfluidic devices for biological assays. ${ }^{36}$

One of the key benefits of confining concentration gradients within such stagnation flow is the ability to maintain a steady-state concentration gradient at low shear stress conditions. In order to test the time stability of the concentration gradient, we observed the concentration gradients over a period of 2 hours. Image slices of the fluorescein gradient along line B'-B” were extracted from a sequential stack of images taken every 18 seconds. These slices were then appended onto one another to illustrate the variation in concentration gradients over a duration of 2 hours and they are shown as inset images in Fig. 3D. The insets in Fig. 3D indicate the stable concentration gradients formed at different flow rates, highlighting no instabilities in concentration gradients formed under stagnation flow confinement.

\section{Hydrodynamic landscaping of combinational concentration species}

In stagnation flow confinement of concentration gradients, hydrodynamic positioning of the stagnation flow (and entrained concentration gradients) was achieved by varying the flow rate ratios between $\left(Q_{1}=Q_{4}=Q_{1,4}\right)$ and $\left(Q_{2}=Q_{3}=Q_{2,3}\right)$ (flow rate annotations follow that shown in Fig. 1C). Varying the flow rate ratio in this manner allows positioning the stagnation flow along the compressional flow axis (the $y$-axis as defined in Fig. 2A). In order to characterize the movement of the stagnation flow with respect to the flow rate ratio, we scaled the flow rates by introducing a non-dimensionalized flow factor, $\tilde{f}$ where $\tilde{f}=\left(Q_{1,4}-Q_{2,3}\right) /\left(Q_{1,4}+Q_{2,3}\right)$.

Hydrodynamic positioning of the stagnation point was performed by actuating the valve pairs $V_{1}=V_{2}=V_{1,2}$ or $V_{3}=V_{4}=V_{3,4}$. When pressure was applied to the control channel, the valve membrane deflected and reduced the flow within the stagnation chamber; valving configurations are shown in Fig. 1C. During valve actuation, the inlet flow rate is adjusted by the extent of valve actuation (or the valve actuation 
pressure). Supplementary Fig. S3 shows the family of actuation curves that were used to adjust inlet flow rates ranging from $Q=50 \mathrm{n} \mathrm{s}^{-1}$ to $1000 \mathrm{nl} \mathrm{s}^{-1}$. Programming the actuation pressure of the valve pairs adjusts the flow rates into the stagnation flow chamber and produces the desired flow factor for dynamic localization of the stagnation point. Videos capturing stagnation point positioning showed that flow rate ratios efficiently could translate the stagnation point along the $y$-axis with stabilization durations of $\sim 30 \mathrm{~ms}$ or less (see supplementary video, video 1). Stagnation point positions were located by PIV mapping of the chamber as the flow factor was adjusted. Fig. 4A shows that flow factor adjustment results in change of the stagnation point along with its surrounding velocity gradient. In Fig. $\mathbf{4 B}$, stagnation point positions were quantified from the velocity maps and plotted with respect to the flow factor, $\tilde{f}$. Along the $y$-axis, the positioning of the stagnation point can be predicted by a linear relationship such that $\tilde{y}=2.56 \tilde{f}$. Numerical data (dotted line plot in Fig. 4B) agree well with the experimental data to within 2.6\%. By controlling the flow factor, we were able to control and position combinational concentration gradients of fluorescein (green) and Rhodamine 6G (red) dyes with respect to stagnation point location (shown in Fig. 5). Videos of the dynamic positioning of entrained combinational concentration gradients (see supplementary video 2) also illustrate how the MCS moves and stabilizes combinational gradients in a smooth manner, allowing real-time adjustment to be made to combinational concentration landscapes across a wide chamber.

\section{Conclusions}

In this study, we have presented a low flow shear technique of confining combinational and dynamically tunable concentration gradients across diminishing velocity gradients in stagnation flow. Tuning the inlet flow rates, binary or combinational concentration gradients formed across the stagnation point can be hydrodynamically positioned in the chamber. Based on our device design, velocity wells across the stagnation point can effectively balance convective flow and diffusion of concentration species within the chamber, creating a permanently sustained concentration gradient. In addition, it has been demonstrated that the MCS is able to dynamically establish concentration gradients while maintaining low in-chamber shear stresses. While prior gradient generators have demonstrated the reduction of shear flow through the addition of shear relief structures like expansion chambers, membranes or gels, these structures in turn hamper the speed at which concentration gradients can be tuned or moved. This use of velocity wells in stagnation flow addresses the mass transport problem of quickly moving binary and combinational concentration gradients, while inducing low shear flow. Furthermore, such a gradient generation scheme can be used to quickly stabilize rapidly decaying gradients of small molecules while maintaining low in-chamber shear stresses, surpassing the saturation limitations of gel-based low shear gradient generators. The device structure should be well suited for biological studies involving intercellular communication ${ }^{37}$ of bacteria ${ }^{38,} 39$ and 
neutrophils ${ }^{40}$ as concentration gradients can be rapidly targeted to or away from cells of interest, based on real-time experimental observations. Furthermore, it would be interesting to expose adherent cells at the stagnation point to competing and overlapping chemical gradients so as to gain insight into hierarchical decision making processes that occur during intercellular communication and chemotaxis. We do note, however, that the application of stagnation flows to control concentration landscapes may not be suitable for non-Newtonian or viscoelastic solutions as such solutions exhibit flow instabilities under certain circumstances. ${ }^{41,42}$ Nonetheless, our MCS device provides a direct method to hydrodynamically focus and move combinational concentrations on-demand, providing a novel approach to achieving low shear stress combinational gradients in a two-dimensional landscape.

\section{Acknowledgements}

This work was supported by the Agency for Science, Technology and Research (A*STAR), Singapore.

\section{References}

E. M. Johnson-Chavarria, U. Agrawal, M. Tanyeri, T. E. Kuhlman and C. M. Schroeder, Lab Chip, 2014, 14, $2688-2697$.

N. Nève, S. Kohles, S. Winn and D. Tretheway, Cell. Mol. Bioeng., 2010, 3, 213-228.

Y. N. Young and M. J. Shelley, Phys. Rev. Lett., 2007, 99, 058303.

T. T. Perkins, D. E. Smith and S. Chu, Science, 1997, 276, 2016-2021.

R. Dylla-Spears, J. E. Townsend, L. Jen-Jacobson, L. L. Sohn and S. J. Muller, Lab Chip, 2010, 10, 1543-1549.

M. Tanyeri and C. M. Schroeder, Nano Lett., 2013, 13, 2357-2364.

M. Tanyeri, M. Ranka, N. Sittipolkul and C. M. Schroeder, Lab Chip, 2011, 11, 1786-1794.

R. Probst, Z. Cummins, C. Ropp, E. Waks and B. Shapiro, IEEE Control Syst. Mag., 2012, 32, 26-53.

W. M. Deen, Analysis of transport phenomena, Oxford University Press, New York, 1998.

G. I. Taylor, Proc. R. Soc. B, 1934, 146, 501-523.

J. Deschamps, V. Kantsler, E. Segre and V. Steinberg, Proc. Natl. Acad. Sci. U.S.A., 2009, 106, 11444-11447.

R. J. Poole, M. A. Alves and P. J. Oliveira, Phys. Rev. Lett., 2007, 99, 164503.

S. D. Hudson, F. R. Phelan, M. D. Handler, J. T. Cabral, K. B. Migler and E. J. Amis, App. Phys. Lett., 2004, 85, 335-337.

S. J. Haward, PLoS ONE, 2014, 9, e92867.

A. Nakajima, S. Ishihara, D. Imoto and S. Sawai, Nat. Commun., 2014, 5.

A. G. G. Toh, Z. P. Wang, C. Yang and N.-T. Nguyen, Microfluid. Nanofluidics, 2014, 16, 1-18.

D. Ahmed, H. S. Muddana, M. Lu, J. B. French, A. Ozcelik, Y. Fang, P. J. Butler, S. J. Benkovic, A. Manz and T. J. Huang, Anal. Chem., 2014, 86, 11803-11810.

I. Dupin, M. Dahan and V. Studer, J. Neurosci., 2013, 33, 17647-17655.

A. Shamloo, N. Ma, M. M. Poo, L. L. Sohn and S. C. Heilshorn, Lab Chip, 2008, 8, 1292-1299.

J. Shemesh, I. Jalilian, A. Shi, G. Heng Yeoh, M. L. Knothe Tate and M. Ebrahimi Warkiani, Lab Chip, 2015.

N. Bhattacharjee, N. Li, T. M. Keenan and A. Folch, Integr. Biol. (Camb.), 2010, 2, 669-679.

X. Gao, X. Zhang, H. Xu, B. Zhou, W. Wen and J. Qin, Biomicrofluidics, 2014, 8, 052008.

H. Xu, M. M. Ferreira and S. C. Heilshorn, Lab Chip, 2014, 14, 2047-2056.

T. Kim, M. Pinelis and M. M. Maharbiz, Biomed. Microdevices, 2009, 11, 65-73.

Y. Xia and G. M. Whitesides, Annu. Rev. Mater. Sci., 1998, 28, 153-184.

T. Thorsen, S. J. Maerkl and S. R. Quake, Science, 2002, 298, 580-584.

M. A. Unger, H.-P. Chou, T. Thorsen, A. Scherer and S. R. Quake, Science, 2000, 288, 113-116.

C. A. Schneider, W. S. Rasband and K. W. Eliceiri, Nat. Meth., 2012, 9, 671-675.

W. Thielicke and E. J. Stamhuis, J. O. R. S., 2014, 2, 30.

J. Wang, N. Douville, S. Takayama and M. ElSayed, Annu. Biomed. Eng., 2012, 40, 1862-1873.

Z. Li, S. Y. Mak, A. Sauret and H. C. Shum, Lab Chip, 2014, 14, 744-749.

B. Mosier, J. Molho and J. Santiago, Exp. Fluids, 2002, 33, 545-554.

P. O. Gendron, F. Avaltroni and K. J. Wilkinson, J. Fluoresc., 2008, 18, 1093-1101.

S. J. Haward, M. S. N. Oliveira, M. A. Alves and G. H. McKinley, Phys. Rev. Lett., 2012, 109, 128301.

R. G. Bacabac, T. H. Smit, S. C. Cowin, J. J. Van Loon, F. T. Nieuwstadt, R. Heethaar and J. Klein-Nulend, J. Biomech., $2005,38,159-167$.

P.-H. Huang, C. Y. Chan, P. Li, N. Nama, Y. Xie, C.-H. Wei, Y. Chen, D. Ahmed and T. J. Huang, Lab Chip, 2015.

F. Guo, J. B. French, P. Li, H. Zhao, C. Y. Chan, J. R. Fick, S. J. Benkovic and T. J. Huang, Lab Chip, $2013,13,3152-3162$.

P. P. Lele, B. G. Hosu and H. C. Berg, Proc. Natl. Acad. Sci. U.S.A., 2013, 110, 11839-11844.

T. L. Min, P. J. Mears, I. Golding and Y. R. Chemla, Proc. Natl. Acad. Sci. U.S.A., 2012, 109, 9869-9874.

D. Irimia, G. Balazsi, N. Agrawal and M. Toner, Biophys J, 2009, 96, 3897-3916.

P. E. Arratia, C. C. Thomas, J. Diorio and J. P. Gollub, Phys. Rev. Lett., 2006, 96, 144502.

S. J. Haward and G. H. McKinley, Phys. Fluids, 2013, 25, 083104. 


\section{List of figures}
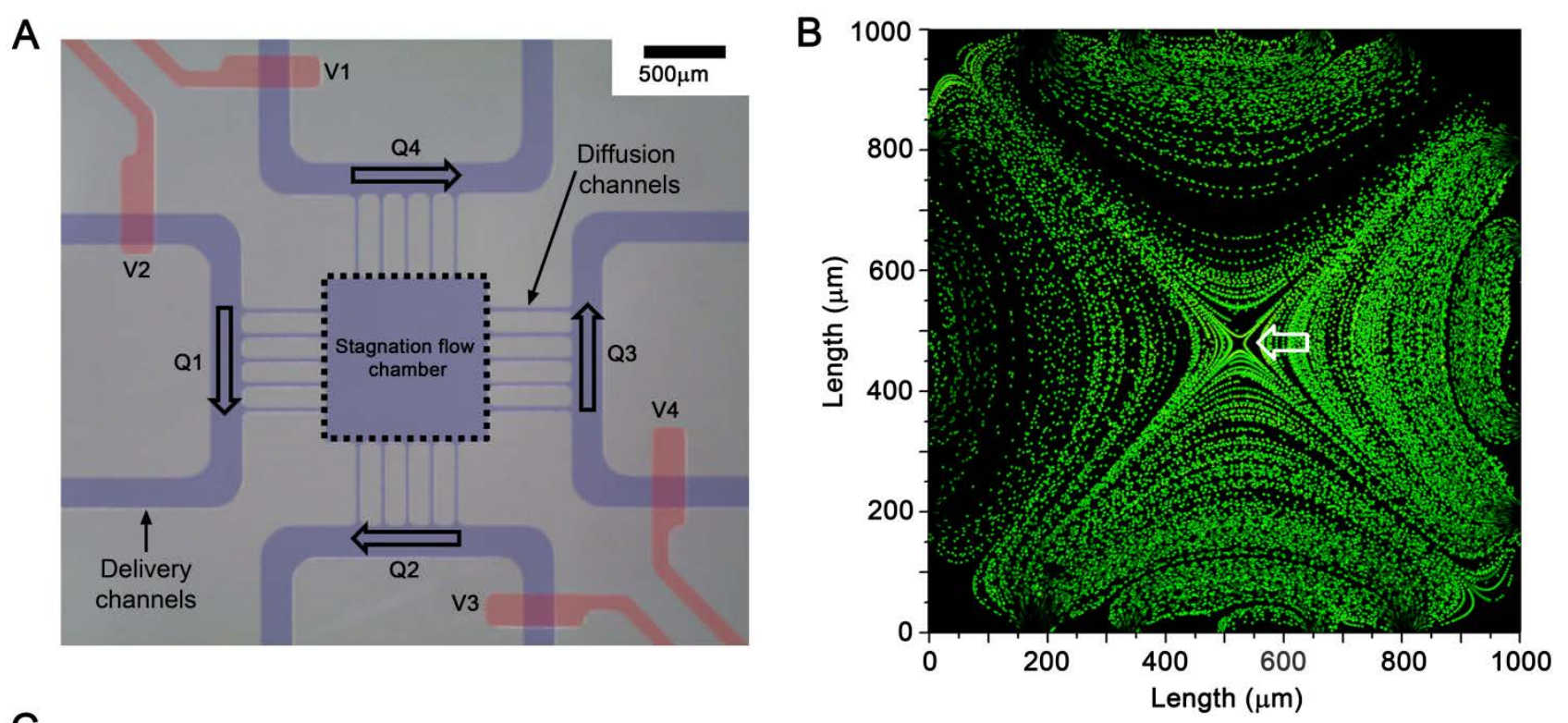

C

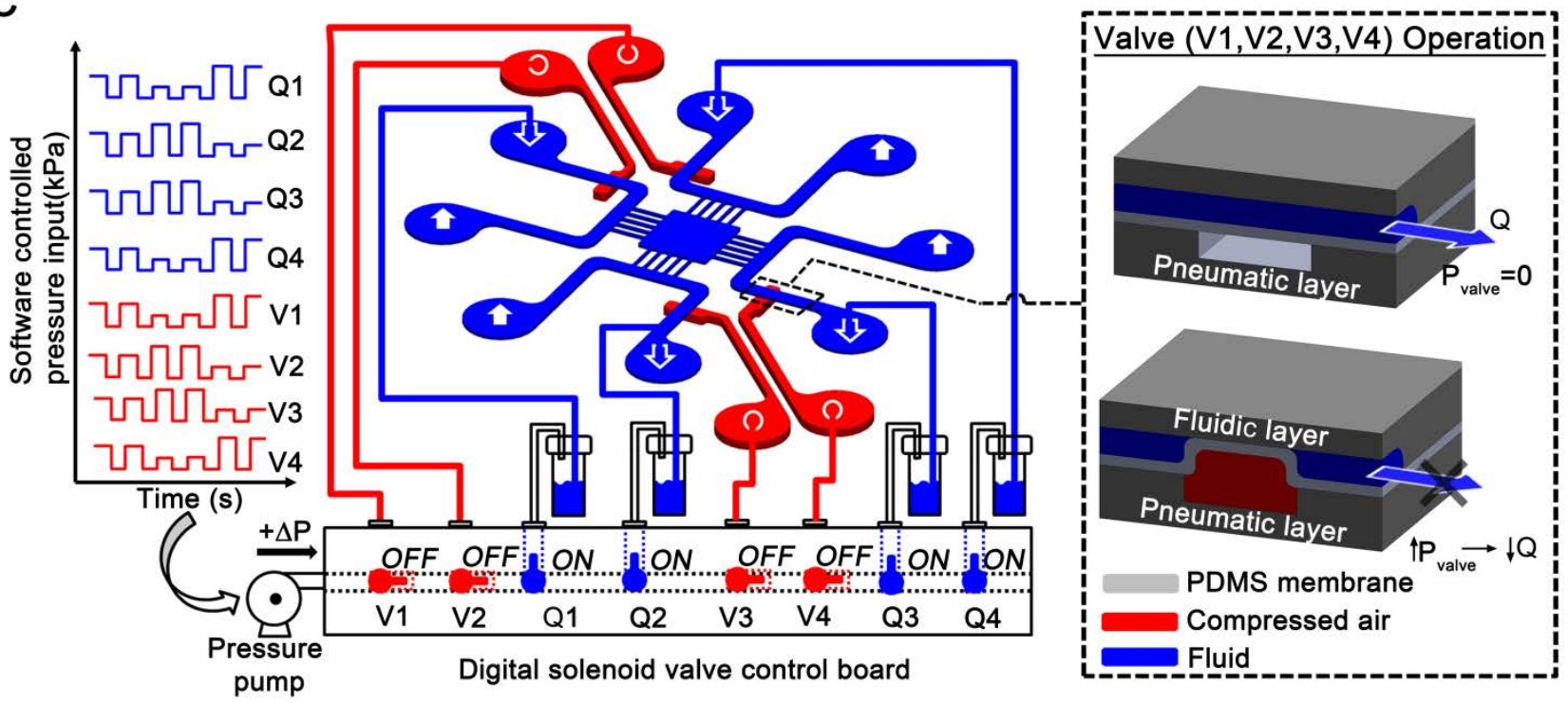

Fig. 1 The modified cross-slot (MCS) device and experiment setup for controlling stagnation flow concentration gradients.(A) Microscope image of the modified cross-slot gradient device and (B) Tracer beads highlight the formation of a stagnation point (indicated by the white arrow) in the chamber centre. (C) Pressure control utilized in experiments to manipulate stagnation flow. Right inset shows the fluidic layer (upper) and the valve layer (lower) cross-section view in operation. During valve operation, $\mathrm{P}_{\text {valve }}>0$ when compressed air is introduced into the pneumatic layer, reducing flow rate in the fluidic layer above. 


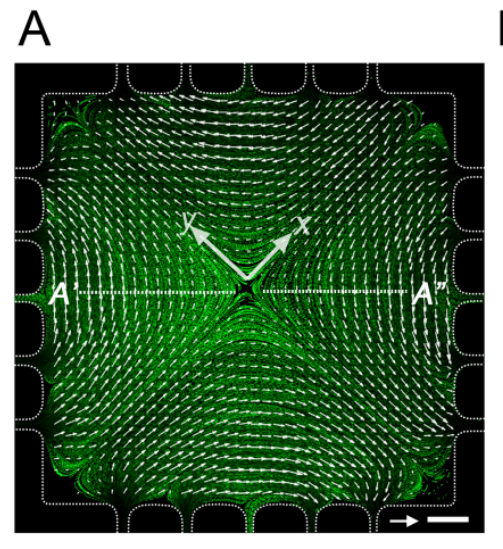

D $\quad Q=100$ n s $^{-1}$
B

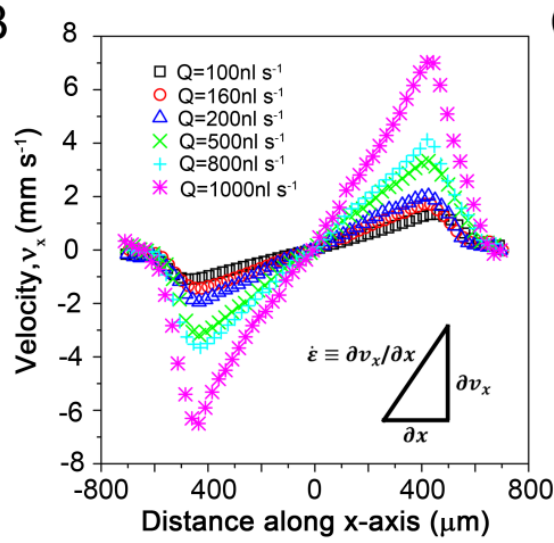

$Q=500 n l s^{-1}$
C

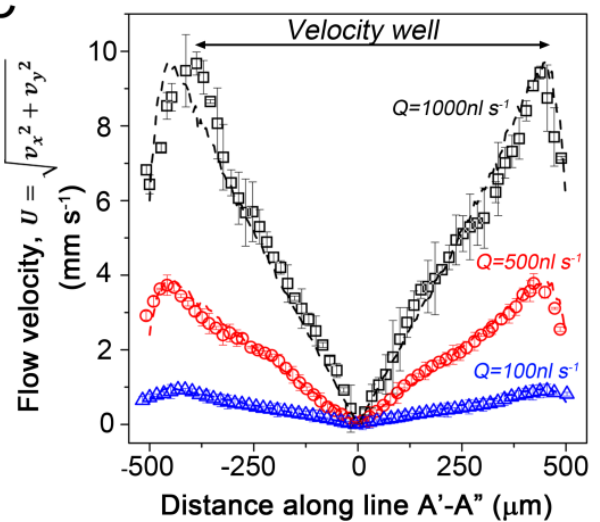

$Q=1000 n ~ s^{-1}$
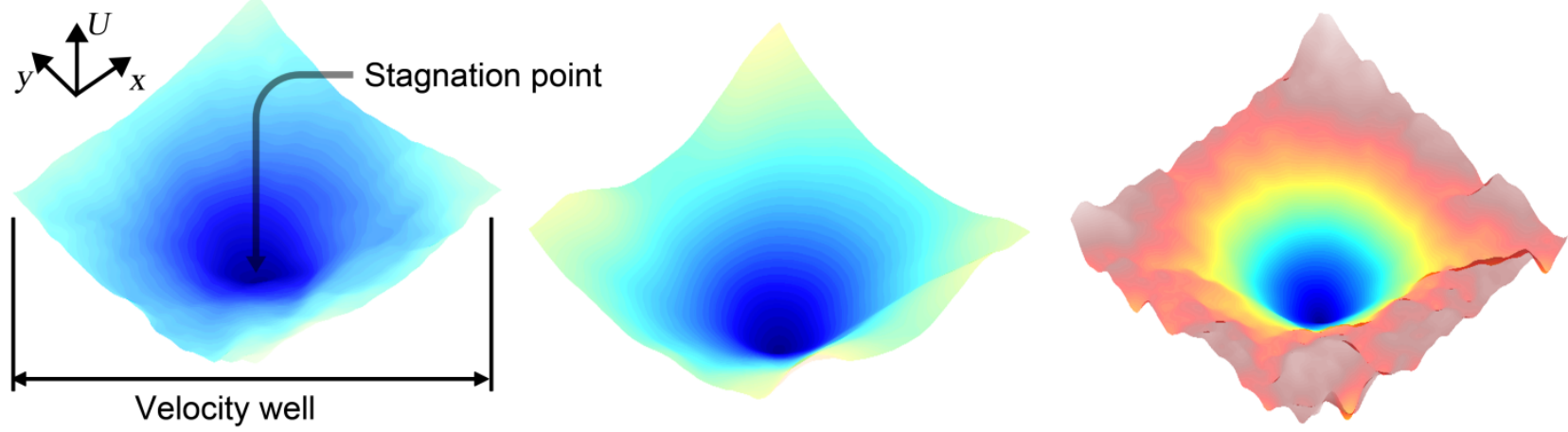

Fig. 2 (A) Vector mapping of flow velocities within the stagnation chamber $\left(Q=100 \mathrm{nl} \mathrm{s} \mathrm{s}^{-1}\right)$. Arrow scale indicates velocity vector strength of $2 \mathrm{~mm} \mathrm{~s}^{-1}$. (B) Variation of $x$-component velocity, $v_{x}$ along the $x$-axis within the vicinity of the stagnant point. Inset in figure shows how the linear flow strain rate, $\dot{\varepsilon}$ is calculated from the velocity profile. (C) Comparison between experimental and numerical velocity profiles extracted along line A'-A". (D) 2D velocity maps of flow gradients surrounding the stagnation point at flow rates of $100 \mathrm{nls}^{-1}, 500 \mathrm{nls}^{-1}$ and $1000 \mathrm{nls}^{-1}$. Contour map for velocity magnitudes ranges from $0 \mathrm{~mm} \mathrm{~s}^{-1}$ (blue) to 10 $\mathrm{mm} \mathrm{s}^{-1}$ (red). 

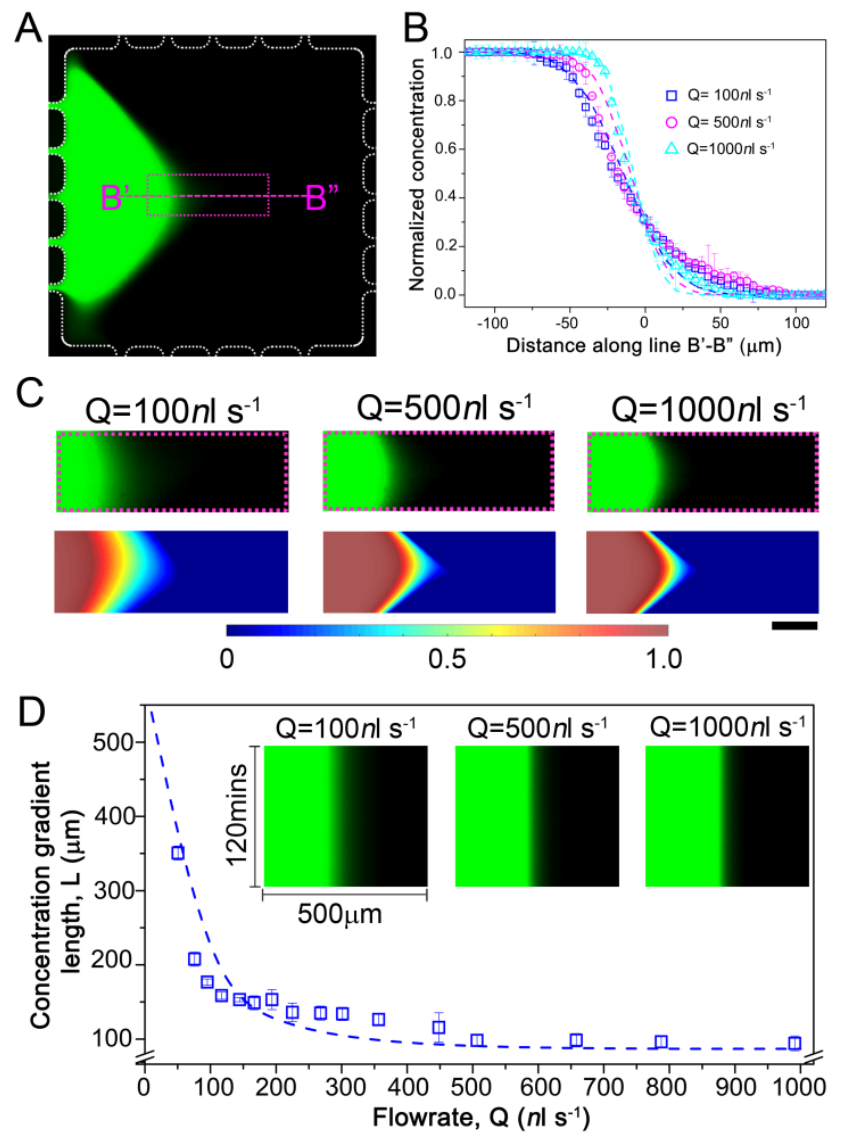

Fig. 3 (A) Binary source fluorescein gradient. (B) Concentration gradient profile along line B'-B” for three different flow rates varying from $100 \mathrm{nls}^{-1}$ to $1000 \mathrm{nls}^{-1}$. (C) $2 \mathrm{D}$ concentration contours comparing experimental (upper) and numerical (lower) concentration gradient formation. Numerical colour map for normalized concentration ranges from 0 (blue) to 1 (red). Scale bar on the bottom right represents $100 \mu \mathrm{m}$ for all images in (C). (D) Effect of flow rate on concentration gradient length, L. (Inset) Time slices of nonsaturating and stable concentration gradients over 2 hours. In plots (B) and (D), lines represent numerical simulation results.

A
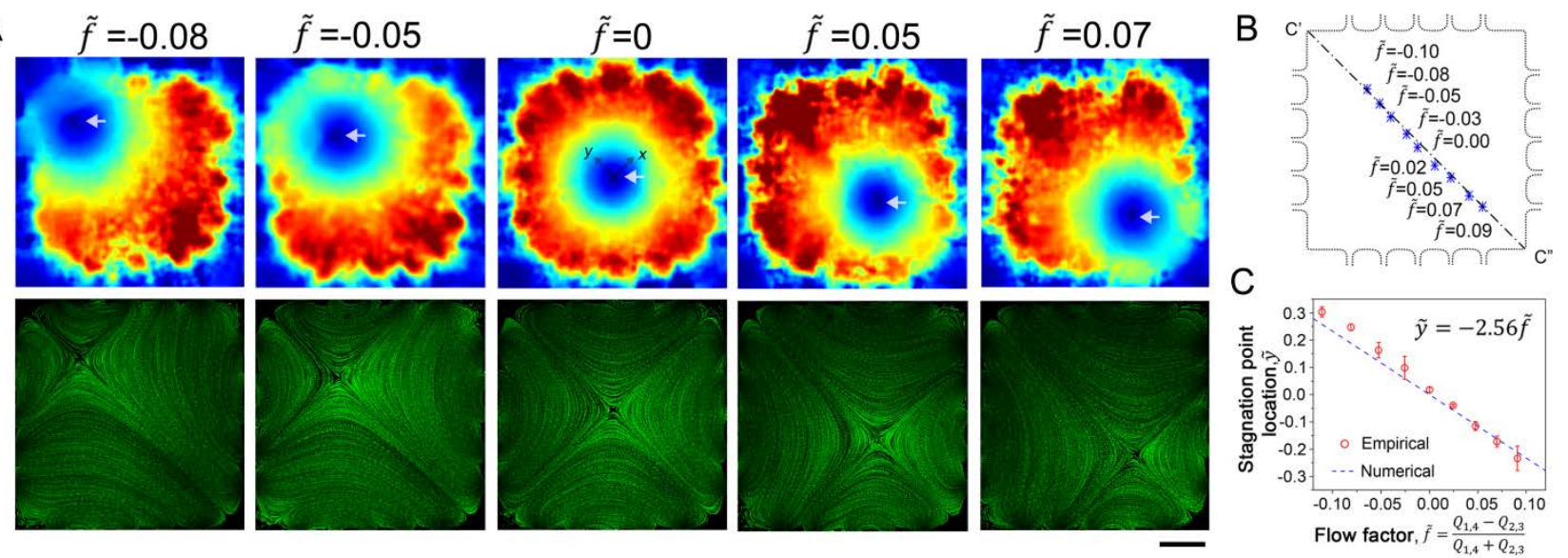
Fig. 4 (A) 2D velocity maps (top) and streakline images (bottom) highlighting the variation of the stagnation point with the non-dimensionalized flow factor, $\tilde{f}$. Circular velocity wells are present around the stagnation point as they are moved across the chamber along line C'-C”. White arrows indicate the stagnation point location. Scale bar on bottom right represents $200 \mu \mathrm{m}$. (B) Locational map of stagnation point translation along line C'-C”. (C) Characterization plot of the stagnation point location, $\tilde{y}$ with respect to flow factor, $\tilde{f}$. The stagnation point location, $\tilde{y}$ is scaled using the length of line C'-C” such that $\tilde{y}=\frac{y}{C^{\prime}-C^{\prime \prime} \text { length }}$.

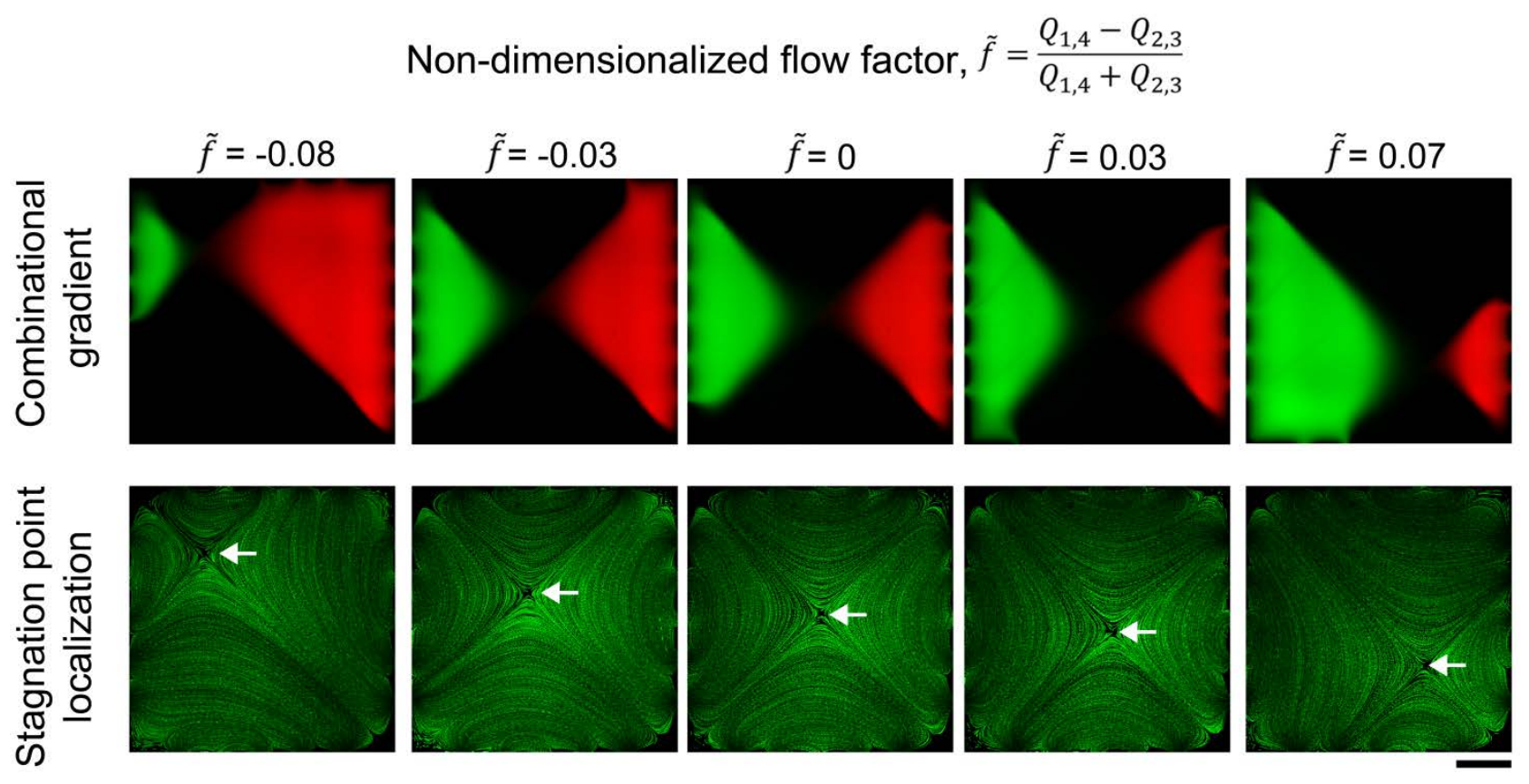

Fig.5 (Upper) Confinement of fluorescein and rhodamine 6G overlapping concentration gradients as stagnation flow is tuned with respect to the flow factor. (Lower) Localization of stagnation point with flow factor adjustment. Arrows point to stagnation point location for each non-dimensionalized flow factor, $\tilde{f}$. Scale bar on the bottom right represents $200 \mu \mathrm{m}$ for all images. 